\title{
LEVANTAMENTO FLORÍSTICO NA ESTAÇÃO ECOLÓGICA DO TRIPUÍ, OURO PRETO, MG ${ }^{1}$
}

\author{
Gilberto Pedralli ${ }^{2}$ \\ Valéria Lúcia de Oliveira Freitas \\ Sylvia Therese Meyer ${ }^{2}$ \\ Maria do Carmo Brandão Teixeira \\ Ana Paula Santos Gonçalves
}

Recebido em 9/1/97. Aceito em 28/12/97

\begin{abstract}
RESUMO - (Levantamento florisitico na Estação Ecológica do Tripuí, Ouro Preto, MG). Realizou-se o levantamento florístico na Estação Ecológica do Tripui, Ouro Preto, MG $\left(43^{\circ} 34^{\prime} 33^{\prime \prime}\right.$ W e $\left.20^{\circ} 23^{\prime} 45^{\prime \prime} \mathrm{S}\right)$. O clima da região é do tipo mesotérmico, com inverno seco (Cwb sg. Köppen) e com temperaturas médias oscilando entre $14^{\circ} \mathrm{e} 19^{\circ} \mathrm{C}$. A Estação apresenta como principais tipos vegetacionais as florestas mesófilas estacionais, o cerrado, a vegetação aquática (brejos, lagoa artificial e córregos) e as formações sucessionais ('candeial'). Para o levantamento florístico utilizou-se o método de parcelas e coletas por trilhas e caminhos, sendo identificadas 101 familias, 242 gêneros e 462 espécies. As familias que apresentaram maior riqueza específica foram Asteraceae $(10,82 \%)$, Melastomataceae $(8,22 \%)$, Myrtaceae $(7,14 \%)$ e Rubiaceae $(4,76 \%)$.
\end{abstract} Palavras-chave: florística, inventário, Estação Ecológica do Tripui, Ouro Preto, Minas Gerais,
fanerógamas, pteridófitas

\begin{abstract}
Floristic inventory in the Tripui Ecological Station, Ouro Preto, MG). A floristic inventory

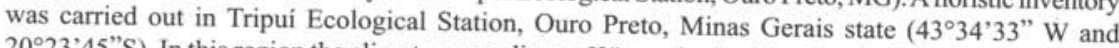
$\left.20^{\circ} 23^{\prime} 45^{\prime \prime} \mathrm{S}\right)$. In this region the climate, according to Köppen's classification, is Cwb, i.e., with a mild summer and a dry winter. The main vegetation types identified were semideciduous mesophytic forests, cerrado, aquatic vegetation ('brejos', artificial lake and creeks) and secondary formations ('candeial'), Through the floristic inventory in plots, trails and ways were identified 101 families, 242 genera and 462 species. Asteraceae $(10,82 \%)$, Melastomataceae $(8,22 \%)$, Myrtaceae $(7,14 \%)$ and Rubiaceae $(4,76 \%)$ were the families with the greatest specific richness.
\end{abstract}

Key words: floristic, inventory, Tripui Ecological Station, Ouro Preto, Minas Gerais State, phanerogamous, pteridophytes

\footnotetext{
Trabalho apresentado no XLV Congresso Nacional de Botânica, São Leopoldo, RS. Auxilios: FNMA e FAPEMIG

Setor de Recursos da Terra, Fundação Centro Tecnológico de Minas Gerais, C.P. 2306, CEP 31170-000,
} 


\section{Introdução}

A Estação Ecológica do Tripuí (E.E.T.) localiza-se no município de Ouro Preto, sudeste da zona mais industrializada e densamente povoada do Estado de Minas Gerais, denominada Zona Metalúrgica. A região em que se insere a E.E.T. recebeu também da UNESCO, a titulação de Reserva da Biosfera da Mata Atlântica (FEAM 1995).

No mapa fitogeográfico de Martius (1858), a região de Ouro Preto encontra-se entre as regiões denominadas Oreades e Dryades. Veloso (1966) enquadra a região na área das "florestas estacionais semideciduais". Em 1991, este autor afirmou que o "conceito deste tipo de vegetação está condicionado pela dupla estacionalidade climática, uma tropical, com época de intensas chuvas de verão, seguida por estiagens acentuadas, e outra subtropical, sem período seco, mas com seca fisiológica provocada pelo intenso frio de inverno, com temperaturas médias inferiores a $15^{\circ} \mathrm{C}$ ".

No mapa dos Domínios Morfoclimáticos da América do Sul, Ab’Saber (1977) inclui a região na área de transição entre os "Domínios da Floresta Atlântica e dos Cerrados".

Segundo Ferri (1980), a região situa-se no Domínio das "Florestas Costeiras ou Atlântica", mostrando em sua composição florística algumas espécies mais representativas, como as quaresmeiras ou manacás-da-serra (Tibouchina spp.), os ipês de flores roxas, amarelas ou brancas (Tabebuia sp.) e as várias espécies de Cassia spp. e Vochysia sp. (pau-tucano) de flores amarelas. Dentre os fetos arborescentes cita Cyathea spp. e Alsophila spp.

Para Eiten (1983), a região se insere na Floresta Atlântica, que apresenta manchas de campos rupestres e campos montanos intercalados e, para o IBGE (1992), a região de Ouro Preto situa-se no Domínio da formação "Floresta Estacional Semidecidual Submontana".

$\mathrm{Na}$ área da E.E.T. foram realizados, anteriormente, levantamentos florísticos não sistematizados (Badini 1985, 1988). Na primeira lista apresentada em 1985, foram identificadas 57 famílias, 142 gêneros e 208 espécies e na segunda lista , apresentada em 1988, houve um incremento significativo no número de gêneros e espécies, tendo sido identificadas 44 famílias, 160 gêneros e 242 espécies.

O presente trabalho, que é parte de um projeto mais abrangente (CETEC 1996), teve como objetivo o levantamento florístico no âmbito da E.E.T.

\section{Material e métodos}

A E.E.T., criada em 1978 para proteger o habitat do Peripatus acacioi, está situada no município de Ouro Preto, aproximadamente a $100 \mathrm{~km}$ de Belo Horizonte, entre os meridianos $43^{\circ} 34^{\prime} 33^{\prime \prime}$ Long. W e os paralelos $20^{\circ} 23$ ' $45^{\prime \prime}$ Lat. S, correspondendo à porção inferior da sub-bacia do córrego Botafogo. Possui área de 337,0 ha, está localizada entre 1.280 e 1.450 metros de altitude e se limita, geologiacamente, ao norte e ao nordeste por rochas xistosas da Formação Sabará e ao sul e ao sudeste por filitos grafitosos da Formação Barreiro. Os solos são desenvolvidos sobre material litológico atribuído ao Grupo Minas, constando essencialmente de xistos, filitos e, bem menos freqüentemente, influenciado por quartzitos. Ocorre em relevo 
muito movimentado (forte ondulado e montanhoso), com declividades dominantes quase sempre superiores a $25 \%$, atingindo muitas vezes mais de $45 \%$. Apresentam caráter raso (solos Litólicos) ou pouco a mediamente profundo (Cambissolos) com presença de minerais primários facilmente intemperizados. A fertilidade natural é muito baixa, devido aos reduzidos teores de $\mathrm{Ca}, \mathrm{Mg}, \mathrm{K}$ e $\mathrm{P}$ e a acidez é elevada, contendo excesso de Al (CETEC 1996). O clima da região é do tipo Cwb, segundo Köeppen, temperado úmido com inverno seco e verão quente e chuvoso, cujas médias anuais de precipitação variam de 1.450 a $1.800 \mathrm{~mm}$. As temperaturas médias anuais oscilam entre $14^{\circ} \mathrm{e} 19^{\circ} \mathrm{C}$ (Antunes 1986).

O levantamento florístico foi realizado no período de outubro/1991 a novembro/ 1995, em seis parcelas de $10 \times 25 \mathrm{~m}$ e através de caminhadas por trilhas e caminhos da E.E.T. A rede de amostragem e a localização da E.E.T. podem ser observadas na Fig. 1. As áreas foram assim denominadas 1 - Pomar, 2 - Apiário, 3 - Adão, 4 - Fortes, 5 - Esperto e 6 - Trevo. Estas áreas foram selecionadas com base no estádio de desenvolvimento dos 'candeiais', desde as fases iniciais de desenvolvimento, onde Vanillosmopsis erythropappa é a espécie mais importante, até o estádio onde esta espécie praticamente desaparece, na floresta mesófila melhor estabelecida. Além deste, outros dois critérios foram utilizados: a topografia, em diferentes vertentes, e a posição relativa em relação ao ângulo de insolação a que as áreas estão sujeitas.

O material coletado foi herborizado segundo as técnicas convencionais (Mori et al. 1989), e identificado através de morfologia comparada, com o auxílio de bibliografia especializada (Barroso 1978; 1984; 1986; Martius et al. 1840-1906; Reitz 1965-1989), realizando-se comparações com exsicatas depositadas nos Herbários do Departamento de Botânica da Universidade de São Paulo (SPF) e do Instituto de Botânica de São Paulo (SP) e enviando-se materiais para especialistas. Os materiais, após identificação, foram incorporados ao Herbário e Xiloteca do Setor de Recursos da Terra (SAT), da Fundação Centro Tecnológico de Minas Gerais - CETEC (HXBH).

A lista de espécies foi elaborada segundo o sistema de classificação de Cronquist (1988), sendo apresentada em ordem alfabética por famílias, gêneros e espécies, incluindo-se dados sobre hábitos. Para a definição do hábito das espécies foi utilizada a nomenclatura de Font Quer (1977), com as seguintes abreviaturas: AR- Árvore; AB- Arbusto; E- Erva; L- Liana e M- Macrófita aquática (Cook 1974).

Para o cálculo de similaridade entre este e os diferentes trabalhos da literatura, foi utilizado o índice de Sorensen, citado por Magurran (1988), como segue:

$$
\begin{aligned}
& \mathrm{S}=\frac{2 \times \mathrm{j}}{\mathrm{a}} \text { onde } \mathrm{j}=\text { número de espécies comuns a ambas as areas } \\
& \mathrm{a}=\text { número de espécies na área } 1 \\
& \mathrm{~b}=\text { número de espécies na área } 2
\end{aligned}
$$

\section{Resultados e discussão}

Como resultado do levantamento florístico foram identificadas 101 famílias, 242 gêneros e 462 espécies (Tab. 1), expressivo se comparado aos estudos realizados por Badini $(1985,1988)$ na E.E.T. e em florestas mesófilas semidecíduas do Estado de São Paulo (Gibbs \& Leitão Filho 1978; Martins 1979; Mathes 1980; Cavassan et al. 1984; Pagano \& Leitão Filho 1987). 


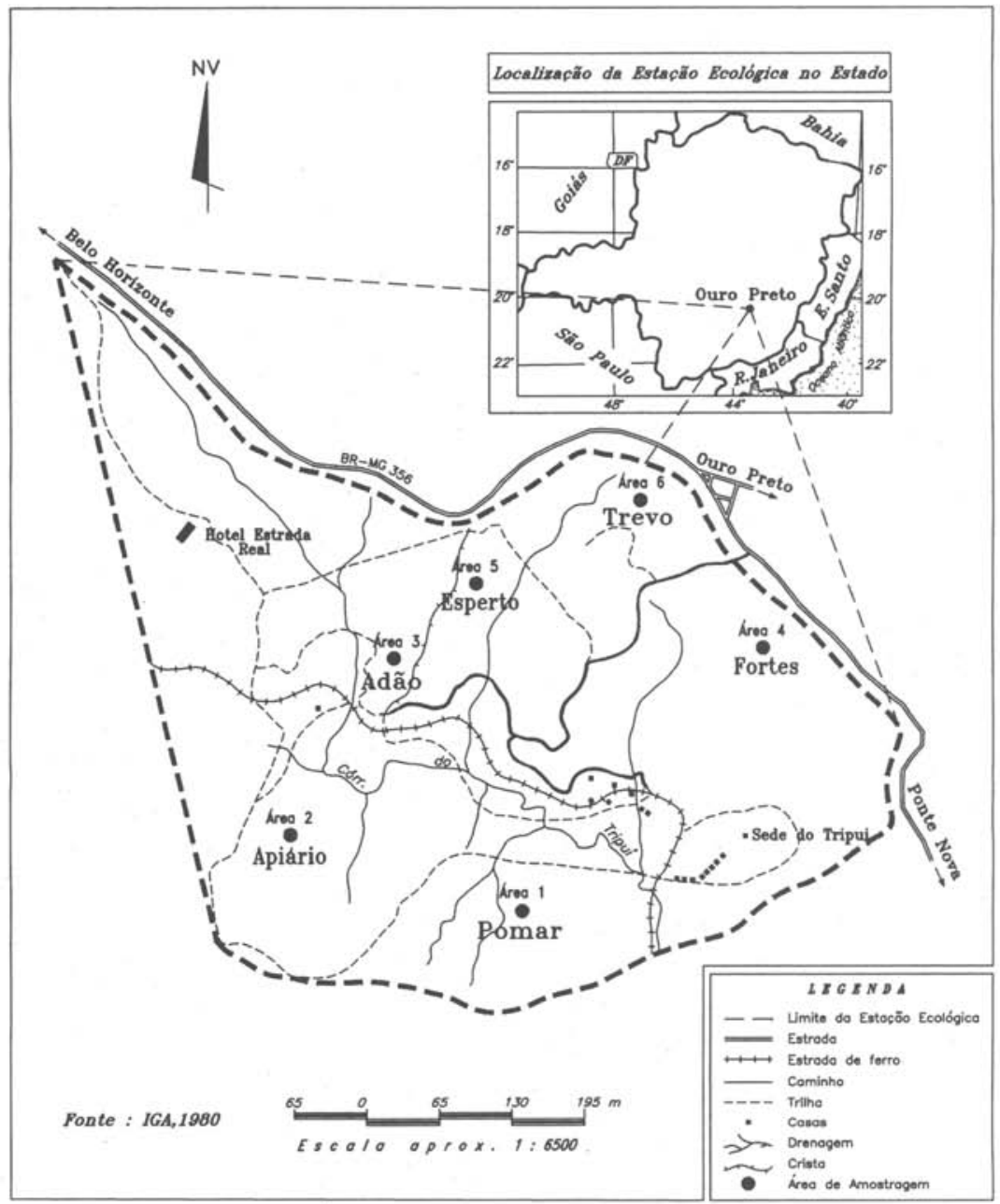

Figura 1. Mapa indicativo das áreas de estudo na Estação Ecológica do Tripui, Ouro Preto, Minas Gerais.

As famílias que apresentaram maior número de espécies foram Asteraceae $(10,82 \%)$, Melastomataceae $(8,22 \%)$, Myrtaceae $(7,14 \%)$ e Rubiaceae $(4,76 \%)$, conforme mostra a Fig. 2. Se consideradas em conjunto, Caesalpiniaceae, Mimosaceae e Fabaceae somaram 6,92\% do total de espécies identificadas (Fig. 2). Essas, somadas às quatro primeiras famílias perfazem $37,86 \%$ do inventário total. Excetuando Asteraceae, estes dados estão de acordo com os levantamentos florísticos em florestas estacionais (mesófilas) da região sudeste do Brasil (São Paulo e Minas Gerais), onde essas famílias aparecem como as mais representativas (Tab. 2) em número de espécies (Cavassan et al. 1984 ; Pagano \& Leitão Filho 1987; Vieira et al. 1989; Rodrigues et al. 1989; Grombone et al. 1990; Vilela et al. 1994; Pagano et al. 1995). 
Tabela 1. Espécies identificadas e respectivos hábitos na Estação Ecológica do Tripuí, Ouro Preto, MG.

Nome Científico / Nome Popular Hábito

\section{ACANTHACEAE}

Mendoncia cf. puberula Mart.

ALSTROEMERIACEAE

Alstroemeria sp.

ANACARDIACEAE

Anacardium occidentale $\mathrm{L}$.

Schinus terebinthifolius Raddi ("aroeirinha")

Tapirira guianensis Aubl. ("peito-de-pombo, pau-pombo")

AR

AR

ANNONACEAE

Guatteria gomeziana St.Hil.

$G$. cf. pohliana Schlecht.

AR

G. sellowiana Schlecht.

AR

G. vilosissima St.Hil.

AR

Xylopia sp. ("pimenta-de-macaco")

AR

AR

$\mathrm{AB}$

APIACEAE (=UMBELLIFERAE)

Centella biflora Nannf.

\section{APOCYNACEAE}

Aspidosperma parvifolium DC.

A. cf. polyneuron Muell.Arg.

\section{AQUIFOLIACEAE}

Ilex aff. conocarpa Reissk.

I. grandii Reissk.

I. retusa Reissk.

I. subcordata Reissk.

I. cf. theezans Mart.

ARALIACEAE

Didymopanax macrocarpum (Cham. et Schlecht.) Seem.

D. morototoni Decne \& Planch.

ARAUCARIACEAE

Araucaria angustifolia (Bert.) O.Kze. ("pinheiro-do-paraná")

ASCLEPIADACEAE

Blepharodum nitidum (Vell.) Macbr.

Calostigma cf. guilleminianum Decne

Ditassa cf. edmundoi Fontella \& Valente

ASTERACEAE (=COMPOSITAE)

Achyrocline satureoides (Lam.) DC. ("marcela")

Achyrocline sp. "marcela"

Ageratum conyzoides $\mathrm{L}$.

E

Baccharis calvescens DC.

E

B. helychrysoides DC.

E

B. mesoneura DC.

E

B. cf. opuntioidea Mart. ex Bak. ("carqueja")

E

B. platypoda $\mathrm{DC}$.

$B$. reticulata DC.

B. cf. tarchonanthoidea DC. ("assa-peixe")

B. trimera DC. ("carqueja")

Baccharis sp. 
Tabela 1 - contiuação

Nome Científico/Nome Popular

Hábito

Bidens pilosa L. ("picão-preto")

$\mathrm{E}$

Eclipta alba Hassk.

Emilia sagitata DC.

Eremanthus incanus Less. ("candeião")

E.cf. sphaerocephalus Bak.

E. amygdalium Lam.

E. buplevifolium DC.

E. cinero-viride Schutlz

E. gaudichaudianum DC.

E. cf. hebecladum DC.

E. intermedium DC.

E. aff. itatiaynensis Hier.

E. aevigatum Lam.

E

E

AR

E

$\mathrm{AB}$

E

E

E. polystachyum DC.

$A B L$

$\mathrm{AB}$

$\mathrm{AB}$

E

E. squarrulosum H. \& A.

$\mathrm{AB}$

$\mathrm{AB}$

E. vautherianum DC.

E

Gochnatia sp.

M. cf. capricornis Rob.

M. cordifolia (L.f.) Willd.

M. hirsutissima DC.

Piptocarpha axillaris (Less.) Bak.

$P$. cf. macropoda Bak.

$P$. aff. oblonga Bak.

$P$. rotundifolia Bak.

Porophyllum cf. lineare DC.

Porophyllum ruderale (Jacq.) Cass.

$\mathrm{AB}$

AR

L

L

L

AR

Symphyopappus polystachyus DC.

Trixis sessinjii DC.

$T$. verbasciformis Less.

Vanillosmopsis erythropappa Schult. Bip. ("candeia")

$\mathrm{AB} / \mathrm{E}$

AR

AR

E

E

E

E

E

Verbesina glabrata Hook. \& Arn.

AR

Vernonia cognata Less.

V. discolor (Spreng.) Less.

$V$. mucronulata Less.

$V$. cf. nitidula Less.

$V$. aff. polyanthes Less.

V. aff. scorpioides (Lam.) Pers.

V. tweediana Bak.

E

E

AR

AR

$\mathrm{AB}$

$\mathrm{AB}$

E

E

\section{BALANOPHORACEAE}

Langsdorffia hypogaea Mart.

BEGONIACEAE

Begonia hispida Schott ex DC. ("begônia")

$B$. cf. reniformis Dryander

E

E

BERBERIDACEAE

Berberis laurina Bellb.

E

BIGNONIACEAE

Distictis sp.

Jacaranda caroba (Vell.) DC. ("jacarandá”)

Lundia nitidula DC.

Pyrostegia venusta Miers (“cipó-São-João")

Stizophyllum sp. 
Tabela 1. contiuação

Nome Científico/ Nome Popular

Hábito

Tabebuia alba (Cham.) Sand.

T. chrysotricha (Mart. ex DC.)Standl. ("ipê-amarelo")

AR

T. vellosoi Toledo ("ipê-amarelo")

AR

\section{BLECHNACEAE}

Blechnum cordatum (Desv.) Hier.

B. regnellianum (Kunze) C.Chr.

B. serrulatum L.C. Rich

E

E

$\mathrm{E}$

BRASSICACEAE (= CRUCIFEARE)

Brassica campestris $\mathrm{L}$.

E

Cardamine bonariensis Bak.

$\mathrm{E}$

Nasturtium sp.

E

BROMELIACEAE

Aechmea bromeliifolia (Rudge) Bak.

E

BUDDLEJACEAE

Buddleja brasiliensis Jacq.f. ex Spreng.

E

BURSERACEAE

Protium heptaphyllum (Aubl.) March. ("almecegueira")

CAMPANULACEAE

Siphocamphylus convolvulaceus G.Don.

E

$S$. corymbiferus Pohl.

$S$. verticilatus (Cham.) G.Don.

S. westinianus (Bilb.) Pohl

CAESALPINIACEAE (= LEGUMINOSAE-CAESALPINIOIDEAE)

Caesalpinia aff. pyramidalis Tul.

Chamaecrista aff. conferta (Benth.) H.S.Irwin \& Barneby

E

E

C. desvauxii (Collad.) Killip.

Copaifera langsdorffii Desf. ("pau-d'óleo")

Senna cana var. hypoleuca (Ness \& Mart.)H.S.Irwin \& Barneby

S. macranthera (Collad.) H.S.Irwin \& Barneby

S. multijuga (L.C.Rich..) H.S.Irwin \& Barneby

S. obtusifolia (L.) F.S.Irwin. \& Barneby

Swartzia pilulifera Benth.

$\mathrm{AB}$

AR

AR

AR

AR

$\mathrm{AB}$

AR

CAPRIFOLIACEAE

Lonicera japonica Thunb.

CELASTRACEAE

Maytenus communis Reissk.

AR

M. gonoclados Mart.

AR

M. pseudocasearia Reissk.

AR

CLETHRACEAE

Clethra scabra Pers.

AR

CLUSIACEAE(=GUTTIFERAE)

Vismia guianensis (Aubl.) Choisy

AR

V. micrantha Mart.

AR

COMBRETACEAE

Combretum sp.

Terminalia aff. glabrescens Mart. 
Tabela 1. contiuação

\begin{tabular}{lc}
\hline Nome Científico/Nome Popular & Hábito \\
\hline COMMELINACEAE & \\
Commelina erecta L. & E \\
Dichorisandra pubescens Mart. & E \\
Tripogandra diuretica (Mart.) Handl. & E \\
CONVOLVULACEAE & \\
Ipomoea aristolochiaefolia G.Don. & L \\
I. cf. franciscana Choisy & L \\
I. purpurea Lam. & L
\end{tabular}

\section{CUNONIACEAE}

Lamanonia ternata Vell. ("guaperê")

\section{CYATHEACEAE}

Trichipteris corcovadensis (Raddi) Copel.

T. gardneri (Hook.) R. M. Tryon

\section{CYPERACEAE}

Ascolepsis brasiliensis (Kunth.) C.B.Clarke

Carex sp.

Cyperus ferax L.C.Rich.

Heleocharis debilis Kunth

\section{DENNSTAEDTIACEAE}

Lindsaea lancea (L.) Bedd.

DILLENIACEAE

Davilla cf. angustifolia St.Hil.

\section{DIOSCOREACEAE}

Dioscorea bulbifera L. ("cará")

D. microbotrya Griseb.

D. scabra H. \& B. in Willd.

\section{DROSERACEAE}

Drosera montana Mart.

\section{DRYOPTERIDACEAE}

Diplazium petersenii (Kunth.) Christ

E

Elaphoglossum subarborescens Ros.

\section{EQUISETACEAE}

Equisetum sp. ("cavalinha")

\section{ERICACEAE}

Gaylussacia fasciculata Gardner

G. salicifolia Cham. et Schlecht. 
Tabela 1. contiuação

Nome Científico/ Nome Popular

ERYTHROXYLACEAE

Erythroxylum aff. engleri Schulz

E. flexuosum Schulz

E. suberosum St. Hil.

\section{EUPHORBIACEAE}

Croton ceanothifolius Baill.

C. floribundus Spreng.

C. urucurana Baill. ("sangra-d'água")

C. widgrenianus Muell.Arg.

FABACEAE (=Leguminosae-Papilionoideae)

Crotalaria anagyroides H.B.K. ("guizo-de-cascavel")

Crudia sp.

Dalbergia brasiliensis Vog.

D. frutescens Britt.

D. variabilis Vog.

D. villosa Benth.

Desmodium incanum (Sw.) DC. ("pega-pega")

D. subsericeum Malme ("pega-pega")

Galactia decumbens Benth.

G. rugosa Benth.

Machaerium aculeatum Raddi

M. villosum Vog.

Macrolobium sp.

Sweetia sp.

FAGACEAE

Castanea sativa Mill.

FLACOURTIACEAE

Abatia americana (Gardner) Eich.

Azara sp.

Casearia decandra Jacq.

C. sylvestris $\mathrm{Sw}$.

Xylosma pseudosalzmanii Sleum.

GENTIANACEAE

Irlbachia pedunculatus (Cham. \& Schlech.) Gilg.

\section{GESNERIACEAE}

Episcia sp.

Gesnera allagophylla Mart.

GLEICHENIACEAE

Dicranopteris linearis (Burm.f.) Underw

Sticherus bifidus (Willd.) Ching

S. pruinosus (Mart.) Ching

HYPOXIDACEAE

Hypoxis decumbens Lam.

E

JUNCACEAE

Juncus dichotomus Ell.

LAMIACEAE (=LABIATAE)

Eriope macrostachya Mart.
$\mathrm{AR}$

AB

AR

AR

AR

AR

E

E

E

E

E

AR

AB

AB

AB

AR

AR

E

$\mathrm{AB} / \mathrm{E}$

AR

AR

AR

AR

E

E

L

AR

AR

AR

AR

R 
Tabela 1 - contiuação

Nome Científico/Nome Popular

Hábito

Eriope sp.

Hyptis carvalhoi Harley

$\mathrm{AB}$

H. lutescens Pohl ex Benth.

$\mathrm{AB} / \mathrm{E}$

H. marrubioides Epl.

E

H. membranacea Benth.

$\mathrm{AB}$

$H$. aff. muricata Schott

$\mathrm{AB}$

$H$. aff. propingua Epl.

E

E

H. suaveolens Poir.

E

LAURACEAE

Cryptocarya aschersoniana $\mathrm{Mez}$ ("canela")

AR

Endlicheria paniculata (Spreng.) Macbride ("canela")

AR

Litsea $\mathrm{sp}$.

Nectandra grandiflora Nees et Mart. ex Nees

AR

$N$. lanceolata Nees et Mart. ex Nees ("canela")

AR

N. leucothyrsus Meissn.

AR

N. puberula Nees ("canela")

N. rigida (H.B.K.) Nees ("canela-amarela")

AR

Ocotea acutifolia (Nees) Mez

AR

O. basicordatifolia Vatt.

AR

O. corymbosa (Meissn.) Mez

AR

O. felix Coe-Teixeira ("canela")

AT

O. macropoda (H.B.K.) Mez

O. nitidula (Nees et Mart. ex Nees) Mez ("canela")

AR

AR

AR

$O$. pulchella Nees et Mart. ex Nees ("canela")

AR

O. spectabilis (Meissn.) Mez ("canela")

O. spixiana (Nees) Mez

AR

AR

AR

LILIACEAE

Gladiolus sp. ("cultivada")

Neomarica rupestris (Rav.) Chukr.

LORANTHACEAE

Struthanthus flexicaulis Mart. ("erva-de-passarinho")

LYCOPODIACEAE

Lycopodiella alopecuroides (L.) Cranfill

E

L. camporum B. Ø1lg \& P.G. Windisch

E

\section{LYTHRACEAE}

Cuphea carthagenensis (Jacq.) Macbride

C. disperma Koehne

C. ingrata Cham. \& Schlecht.

Diplusodon cf. quintuplinervis (Princ. Weuw.) Koehne

D. virgatus Pohl

Lafoensia densiflora Pohl

L. pacari St.Hil.

E

E

E

$\mathrm{AB}$

$\mathrm{AB}$

AR

AR

MALPIGHIACEAE

Banisteriopsis campestris (Juss.) Little

Byrsonima clausseniana Juss.

B. crassa Nied.

B. crassifolia (L.) H.B.K.

$B$. intermedia Juss.

$B$. aff. lancifolia Juss. 
Tabela 1. contiuação

\begin{tabular}{lc}
\hline Nome Cientifico/Nome Popular & Hábito \\
Byrsonima cf. nervosa DC. & $\mathrm{AB}$ \\
B. sericea DC. & $\mathrm{AR}$ \\
B. variabilis Juss. & $\mathrm{AB}$ \\
Heteropteris aceroides Griseb. & $\mathrm{L}$ \\
H. umbellata Juss. & $\mathrm{L}$ \\
Peixotoa cf. paludosa Turcz & $\mathrm{L}$ \\
P. tomentosa Juss. & $\mathrm{L}$ \\
Tetrapteris acutifolia Cav. & $\mathrm{L}$ \\
T. guilleminiana Juss. & $\mathrm{L}$
\end{tabular}

\section{MALVACEAE}

$\begin{array}{ll}\text { Pavonia commutata Garke } & \text { AB }\end{array}$

$\begin{array}{ll}\text { Sida } \text { spp. } & \mathrm{AB}\end{array}$

MELASTOMATACEAE

Acisanthera variabilis Triana $\quad$ E

Cambessedesia espora DC. $\quad$ E

C. hilariana (St. Hil. ex Bonpl.) DC.

$\begin{array}{ll}\text { Lavosiera pectinata Cogn. } & \mathrm{AB}\end{array}$

Leandra aurea Cogn. $\quad \mathrm{AB}$

L. cf. blanchetiana Cogn. $\quad A B$

L. erostata (DC.) Cogn. $\quad A B$

L. foveolata (DC.) Cogn. $\quad A B$

L. glabrata (Bumb.) Cogn. $\quad A B$

L. melastomoides Raddi $\quad$ AR

Marcetia taxifolia (St.Hil. ex Bonpl.) $\quad$ E

Miconia albicans (Sw.) Triana $\quad$ AR

M. chartacea Triana $\quad$ AR

M. corallina Spring $\quad$ AR

M. ibaguensis (DC.) Triana $\quad$ AR

M. ligustroides (DC.) Naud. $\quad$ AR

$\begin{array}{ll}M \text {. paniculata Naud. } & \text { AR }\end{array}$

M. pepericarpa $\mathrm{DC}$. $\mathrm{AR}$

$M$. cf. rimalis Naud. $\quad A B$

$\begin{array}{ll}M \text {. theaezans (Bonpl.) Cogn. } & \mathrm{AR}\end{array}$

$M$. cf. valtherii Naud. $\quad A B$

Microlicia aff. avicularis Mart. $\quad$ E

M. cordata Cham.

M. isophylla DC. $\quad \mathrm{E}$

Ossaea cinnamomifolia Triana $\quad$ E

O. congestiflora (Naud.) Cogn. $\quad$ E

O. coriacea Triana $\quad$ E

Tibouchina canescens Cogn. $\quad$ E

$\begin{array}{ll}\text { T. cardinalis (Bonpl.) Cogn. } & \mathrm{AR} / \mathrm{AB}\end{array}$

T. gracilis (Bonpl.) Cogn.

$\begin{array}{ll}\text { T. martialis (Cham.) Cogn. } & \mathrm{AB} / \mathrm{E}\end{array}$

T. martiusiana DC. $\quad$ E

T. moricandiana Nannd. $\quad$ AB

T. phlogiformis DC. $\quad \mathrm{AB}$

T. sebastianopolitana (Radd.) Cogn. $\quad$ E

Trembleya laniflora Cogn. var. genuina Cogn. $\quad$ E

T. parviflora (Don.) Cogn. $\quad$ E 
Tabela 1. contiuação

Nome Científico / Nome Popular

Hábito

MELIACEAE

Cabralea canjerana (Vell.) Mart. ("cangerana")

AR

Cedrella sp.

Guarea cf. macrophylla Vahl.

Trichilia catigua A.Juss.

AR

AR

AR

MIMOSACEAE (= LEGUMINOSAE-MIMOSOIDEAE)

Dalbergia brasiliensis Vog.

Inga edulis Mart. "ingá"

I. sellowiana Benth.

I. sessilis Mart. "ingá"

Mimosa acerba Benth.

AR

AR

AR

M. calothamnos Mart.

$\mathrm{AB}$

$M$. aff. dolens Vell.

$\mathrm{AB}$

M. pudica $\mathrm{L}$.

E

Pithecelobium cf. avaremoteno (Mart.) DC.

MONIMIACEAE

Mollinedia argyrogyna Perk.

Siparuna apiosyce (Mart.) DC. ("negramina")

\section{MORACEAE}

Brosimum sp.

Ficus sp.

\section{MYRSINACEAE}

Rapanea ferruginea (R. \& P.) Mez ("capororoca")

\section{MYRTACEAE}

Calycorectes psidiiflorus (Bers.) Sobral

Calycorectes sp.

Calyptranthes cf. clusiaefolia Berg.

Campomanesia adamantium (Camb.) Berg.

C. guaviroba DC.

C. cf. sessiliflora (Berg.) Mattos

C. xanthocarpa Berg.

Eugenia calycina Camb.

E. egensis DC.

$E$. involucrata DC.

E. paniculata Banks

$G$. lindeniana Berg.

G. sellowiana Berg.

Gomidesia sp.

Marlieria cannaefolia Berg.

Myrceugenia myrcioides Camb.

Myrceugenia sp.

Myrcia acuminatissima Berg.

M. calycina Camb.

M. crassifolia (Berg.) Kiaersk.

M. dictyophleba (Berg.) Legr.

$M$. intermedia Kiaersk.

M. laruotteana Camb. 
Tabela 1. contiuação

\begin{tabular}{lc}
\hline Nome Científico/Nome Popular & Hábito \\
\hline Myrcia leptoclada DC. & $\mathrm{AR}$ \\
M. linkiana DC. & $\mathrm{AR}$ \\
M. cf. oblongata DC. & $\mathrm{AR}$ \\
M. tomentosa (Aubl.) DC. & $\mathrm{AR}$ \\
Pimenta pseudocaryophyllus Blume & $\mathrm{AR}$ \\
Plinia sp. & $\mathrm{AR}$ \\
Siphoneugena chnoosepala (Kiaersk.) Kransel & $\mathrm{AR}$ \\
S. densiflora Berg. & $\mathrm{AR}$ \\
S. widgreniana Berg. & $\mathrm{AR}$ \\
NYCTAGINACEAE & \\
Guapira moxia (Netto) Lund. & $\mathrm{AR}$ \\
G. opposita (Vell.) Reitz & $\mathrm{AR}$ \\
Neea sp. & $\mathrm{AR}$ \\
OCHNACEAE & \\
Ouratea castanaefolia (DC.) Engl. & $\mathrm{AR}$ \\
Sauvagesia erecta L. & $\mathrm{E}$ \\
S. velloziana (Vand.) Sastre & $\mathrm{E}$ \\
ONAGRACEAE & \\
Ludwigia laruotteana (Camb.) Hara (“cruz-de-malta") & $\mathrm{E}$ \\
L. nervosa Poir. ("cruz-de-malta") & $\mathrm{E}$
\end{tabular}

\section{ORCHIDACEAE}

Cleistes lepida (Rchb.f.) Schltr.

Epidendrum dendrobioides (Lindl.) Pabst

E. denticulatum Barb. Rodr.

Habenaria armata Richenb.

$H$. parviflora Lindl.

Laelia flava Lindl.

Oncidium battemanianum Parm.

Prescotia montana Barb. Rodr.

Zygopetalum mackayi Hook.

Zygopetalum sp.

\section{PASSIFLORACEAE}

Passiflora haematostigma Mart. ex. Mast. ("maracujáa") L

P. speciosa Gardn. ("maracujá")

Passiflora sp.

PIPERACEAE

Piper cernuum Vell.

P. crassinervium H.B.K.

P. lhotzkyanum Kunth

P. lindbergii DC.

P. salicariifolium Kunth

P. tectonifolium Kunth

POACEAE (=GRAMINEAE)

Andropogon bicornis $\mathrm{L}$.

A. leuchostachyus H.B.K.

Bambusa sp.

Digitaria sp. 
Tabela 1. contiuação

Nome Científico/Nome Popular Hábito

Melinis minutiflora Beauv.

Panicum millegrana Poir.

E

Paspalum polyphyllum Nees

E

Setaria geniculata (Lam.) Beauv.

E

Setaria cf. rarifolia Mik. ex Trin.

E

POLYGALACEAE

Polygala extraxilaris Chod.

E

P. laureola St.Hil. \& Moq.

P. paniculata L. ("barba-de-São-Pedro")

E

P. timoutoides Chod.

E

E

\section{POLYGONACEAE}

Coccoloba vellosiana Casar.

E

Polygonum acuminatum H.B.K. ("erva-de-bicho")

L

POLYPODIACEAE

Pleopeltis astrolepsis (Liebm.) Fourm.

Polypodium fraxinifolium Jacq.

E

P. latipes Langsd. \& Fisch.

E

E

\section{PROTEACEAE}

Euplassa incana (Klotzsch) Johnest.

AR

Roupala montana Bak. ("carne-de-vaca")

AR

PTERIDACEAE

Adiantum raddianum $\mathrm{C}$. Presl

Cheilanthes cf. concolor (Langsd. \& Fisch.) R.M. Tryon \& A. F. Tryon

Pityrogramma trifoliata (L.) R.M. Tryon

Pteris vittata $\mathrm{L}$.

E

E

E

E

RHAMNACEAE

Não identificada

AR

\section{ROSACEAE}

Rubus brasiliensis Mart.

$R$. rosaefolius Smith

\section{RUBIACEAE}

Alibertia concolor (Cham.) Schum.

AR

A. sessilis Schum

Amaioua intermedia Mart.

Borreria capitata (R. et P.) DC.

B. cf. tenella Cham. \& Schlecht.

Coccosypselum erythrocephalum Cham. \& Schlecht.

Chomelia pohliana Muell.Arg.

Faramea latifolia DC.

AR

AR

Geophila sp.

Guettarda sp.

Manettia cf. cordifolia Mart.

Palicourea crocea (Sw.) R \& S.

Palicourea tetraphylla Cham. \& Schlecht.

Psychotria capitata R. et $P$. 
Tabela 1 - contiuação

Nome Científico / Nome Popular

Hábito

Psychotria sp.

AR

Remijia ferruginea DC.

AR

Richardia brasiliensis Gomes

$\mathrm{AB}$

Rudgea sp.

RUTACEAE

Zanthoxylum rhoifolium Lam. ("mamica-de-cadela")

SAPINDACEAE

Cupania vernalis Camb. ("camboatá")

AR

Matayba cf. elaeagnoides Radlk.

M. guianensis Aubl.

M. cf. juglandifolia (Camb.) Radlk.

AR

AR

$M$. cf. punctata Radlk.

AR

Paullinia carpopodea Camb.

SAPOTACEAE

Pouteria venosa (Mart.) Baehni

SAXIFRAGACEAE

Escallonia chlorophylla Cham. \& Schlecht.

E. obtusissima St.Hil.

SCHIZAEACEAE

Anemia flexuosa (Sav.) Sw.

E

A. phyllitidis (L.) Sw.

E

A. villosa Willd.

SCROPHULARIACEAE

Buchnera sp.

Esterhazia splendida Mik.

\section{SMILACACEAE}

Smilax brasiliensis Spreng. ("salsa-parrilha")

S. campestris Griseb. ("salsa-parrilha")

L

Bassovia velutina Dun.

Brunfelsia cf. brasiliensis (Spreng.) Smith \& Downs ("manacá")

Cestrum amictum Schlecht.

$\mathrm{AB}$

C. corymbosum Schlecht.

AR

C. laevigatum Schlecht.

AR

C. lanceolatum Miers

AR

C. nocturnum $\mathrm{L}$.

AR

Solanum americanum Mill.

AR

S. aspero-lanatum R. \& P.

E

$S$. bullatum Vell.

$\mathrm{AB}$

S. lycocarpum St.Hil.

$\mathrm{AR}$

$S$. mauritianum Scop.

E

E

SYMPLOCACEAE

Symplocos cf. phaeoclados DC.

THEACEAE

Laplacea acutifolia (Waw.) Kob.

AR

Thea sinensis L. ("chá-cultivado") 
Tabela 1. contiuação

Nome Científico/ Nome Popular

Hábito

THYMELAEACEAE

Daphnopsis fasciculata (Meissn.) Nevl.

AR

TILIACEAE

Triunfetta semitriloba Jacq. ("carrapicho")

E

TURNERACEAE

Turnera hassleriana Urban

ULMACEAE

Celtis brasiliensis Gardn.

AR

C. iguanaea (Jacq.) Sarj.

AR

Trema sp.

AR AR

VERBENACEAE

Aegiphila integrifolia (Jacq.) Jacq.

AR

A. intermedia Mold.

AR

Lantana aff. canescens H.B.K.

$\mathrm{AB}$

L. lilacina Desf.

$\mathrm{AB}$

L. lundiana Schaw.

E

L. undulata Schr.

$\mathrm{AB}$

Vitex cf. cymosa Bert. ex Spreng.

AR

V. sellowiana Cham.

AR

VISCACEAE

Phoradendron crassifolium (Pohl) Eichl.

P. holaxanthum Eichl.

$P$

$\mathrm{P}$

VOCHYSIACEAE

Qualea parviflora Mart.

AR

Vochysia tucanorum Mart. ("pau-tucano")

AR

XYRIDACEAE

Xyris spp. ("botão-de-ouro")

E

WINTERACEAE

Drymis brasiliensis Miers ("casca-de-anta")

$\mathrm{AB}$

ZINGIBERACEAE

Hedychium coronarium Koeng. ("lírio-do-brejo")

Na Tab. 3 pode-se observar, segundo o índice de Sorensen, o percentual de similaridade entre famílias, gêneros e espécies, nos vários estudos realizados em São Paulo, Minas Gerais e neste trabalho. Observa-se que em níveis específico e genérico, apesar de baixas, as maiores similaridades foram obtidas com relação aos estudos de Vilela et al. (1995) e Oliveira Filho et al. (1994), em florestas mesófilas do sul de Minas Gerais.

Pagano et al. (1995), que estudaram uma floresta mesófila em Rio Claro, no Estado de São Paulo, utilizando o índice de Jaccard, afirmam que as florestas mesófilas podem apresentar diferenças florísticas significativas em áreas muito próximas, mesmo utilizando-se métodos de amostragem semelhantes. 


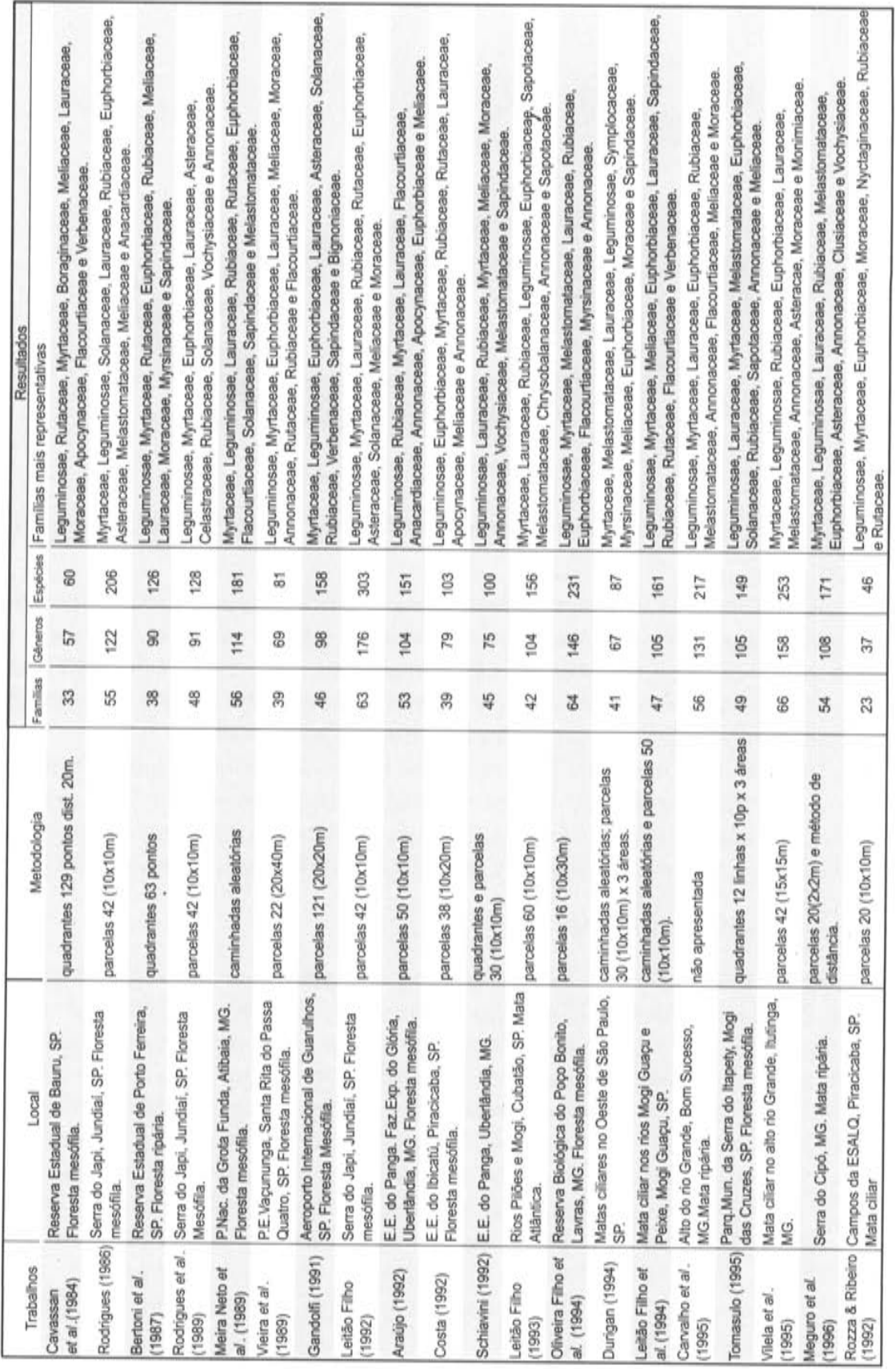

Tabela 2. Levantamento florístico e fitossociológico de espécias arbóreas realizados em diferente áreas florestais na região sudeste do Brasil 


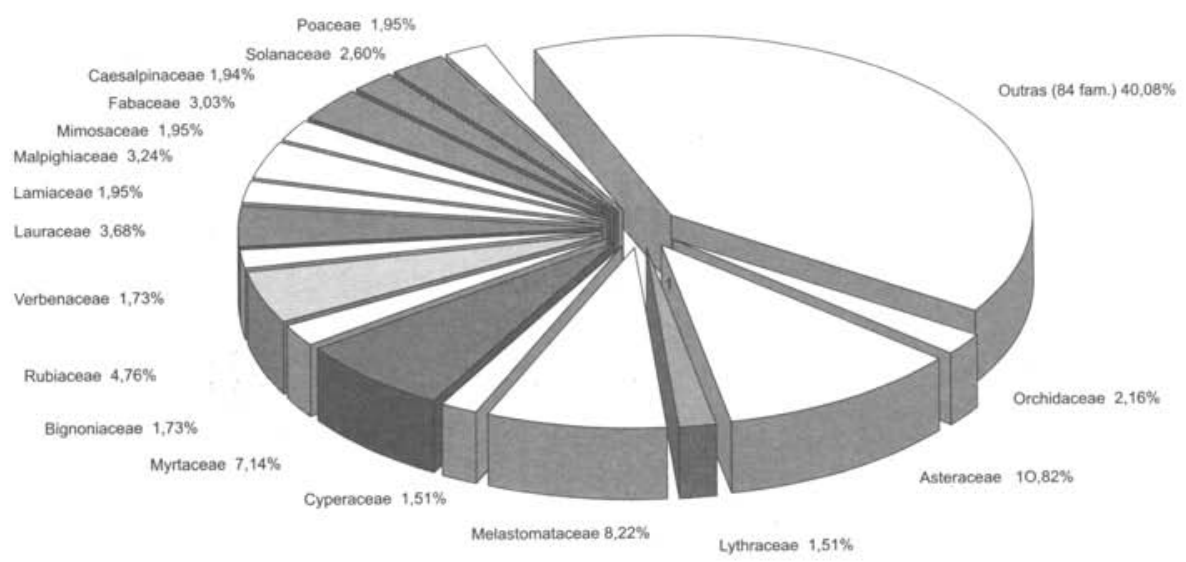

Figura 2. Riqueza especifica por familia no levantamento florístico, da Estação Ecológica de Tripui, Ouro Preto MG.

O grande número de espécies de Asteraceae identificadas e, conseqüentemente, sua alta riqueza específica deve-se principalmente ao estádio sucessional em que se encontra a vegetação da E.E.T. Observa-se a presença de muitas espécies herbáceas invasoras, pertencentes a essa família, que ocorrem nos estádios iniciais de ocupação de áreas perturbadas e que possuem grande plasticidade ambiental.

Os dados do levantamento florístico e as observações de campo permitiram definir-se as seguintes formações: florestas mesófilas estacionais, cerrado, vegetação aquática (brejos, lagoa artificial e córregos), áreas de transição entre esses tipos, áreas com formações sucessionais secundárias (naturais e antrópicas) e áreas cultivadas.

Descrição das principais fisionomias

Florestas Mesófilas - São formações predominantemente arbóreas, silvestres, climáticas, decíduas e semidecíduas, que ocorrem na faixa tropical (FEAM 1995; CETEC 1996). O grau de deciduidade depende da estacionalidade, isto é, da duração do período de seca e também da capacidade de retenção de água pelo solo. Algumas das espécies identificadas nessas florestas: Protium heptaphyllum (Aubl.) March. (almecegueira), Guatteria vilosissima St.Hil. (pindaíba), Tabebuia sp. (ipê-amarelo), Clethra scabra Pers., Piptocarpha cf. macropoda Bak., Vanillosmopsis erythropappa Schul. Bip. (candeia), Vismia guianensis (Aubl.) Choisy, Endlicheria paniculata (Spreng.) Macbride (canela-papagaio), Nectandra lanceolata Nees et Mart. ex Nees (canela), Ocotea spectabilis (Meissn.) Mez (canela), Copaifera langsdorffii Desf. (pau-d'óleo), Dalbergia villosa Benth. (jacarandazinho), Aspidosperma parvifolium DC., Alibertia concolor (Cham.) Schum., Miconia cf. chartacea Triana, Tibouchina canescens Cogn. (quaresmeira), Psychotria cf. sessilis (Vell.) Muell. Arg., Vitex sellowiana Cham., Cabralea cangerana (Vell.) Mart. (cangerana), Rapanea ferruginea (R. et P.) Mez (capororoca), Myrcia leptoclada DC. e Myrcia linkiana DC. 
Tabela 3. Comparativo da riqueza florística de espécies arbóreas, em diferentes áreas florestais na região sudeste, com os resultados obtidos no levantamento florístico na Estação Ecológica do Tripuí, Ouro Preto, MG

\begin{tabular}{|c|c|c|c|c|c|c|c|}
\hline \multirow[b]{2}{*}{ Trabalhos } & \multicolumn{4}{|c|}{ táxons comuns } & \multicolumn{3}{|c|}{ Índice de similaridade de Sorensen } \\
\hline & Famílias & Gêneros & Espécies & $\begin{array}{l}\text { Familias mais } \\
\text { representativas } \\
(\%)\end{array}$ & Espécies & Gêneros & Familias \\
\hline Cavassan et al. (1984) & 26 & 21 & 9 & 40 & 5,9 & 24,7 & 64,2 \\
\hline Rodrigues (1986) & 41 & 55 & 30 & 60 & 16,0 & 53,9 & 85,4 \\
\hline Bertoni et al. (1987) & 26 & 38 & 17 & 40 & 9,1 & 37,4 & 60,5 \\
\hline Rodrigues et al. (1989) & 41 & 55 & 30 & 60 & 16,0 & 53,9 & 85,4 \\
\hline Meira Neto et al. (1989) & 35 & 58 & 36 & 60 & 16,8 & 51,1 & 67,3 \\
\hline Vieira et al. (1989) & 25 & 30 & 11 & 40 & 6,7 & 33,0 & 57,5 \\
\hline Gandolfi (1991) & 33 & 51 & 32 & 70 & 15,9 & 28,3 & 70,2 \\
\hline Leitão Filho (1992) & 42 & 74 & 55 & 50 & 20,0 & 41,2 & 75,7 \\
\hline Araújo (1992) & 34 & 51 & 24 & 40 & 12,1 & 47,0 & 67,3 \\
\hline Costa (1992) & 25 & 37 & 19 & 40 & 10,9 & 38,5 & 57,5 \\
\hline Schiavini (1992) & 29 & 39 & 21 & 50 & 12,1 & 41,5 & 62,4 \\
\hline Oliveira Filho et al. (1994) & 29 & 43 & 15 & 50 & 7,4 & 39,6 & 64,4 \\
\hline Durigan (1994) & 46 & 81 & 50 & 50 & 20,9 & 62,5 & 82,1 \\
\hline Leitão Filho et al. (1994) & 32 & 49 & 26 & 40 & 15,6 & 54,4 & 72,0 \\
\hline Carvalho et al. (1995) & 30 & 47 & 34 & 50 & 16,7 & 43,1 & 63,2 \\
\hline Tomasulo (1995) & 39 & 62 & 43 & 50 & 18,5 & 50,8 & 75,0 \\
\hline Vilela et al. (1995) & 34 & 53 & 30 & 60 & 15,2 & 48,6 & 70,1 \\
\hline Meguro et al. (1995) & 45 & 78 & 58 & 60 & 23,2 & 57,6 & 78,9 \\
\hline Meguro et al. (1996) & 40 & 59 & 48 & 60 & 23,0 & 53,4 & 78,4 \\
\hline Rozza et al. s.d. & 18 & 15 & 7 & 40 & 4,8 & 20,0 & 50,7 \\
\hline
\end{tabular}

Cerrados - São formações savanícolas, campestres, bioestratificadas, onde o estrato herbáceo é dominado por gramíneas. Os cerrados são considerados um complexo vegetacional (Rizzini 1979; Fernandes \& Bezerra 1990) que se destacam pela escleromorfia foliar e pela suberização dos seus elementos florísticos. Entre 40-50\% das espécies do cerrado ocorrem em múltiplas combinações nas suas variações fisionômicas (campo limpo, campo sujo, campo cerrado e cerrado típico). Os limites e interfaces entre essas fisionomias só podem ser corretamente definidos quando se realiza trabalho acurado de levantamento florístico e de análise da sua estrutura. Para a definição fisionômica no âmbito da E.E.T., tomou-se por base comparativa as ilustrações de Eiten (1983). No campo limpo de cerrado foram identificadas Diplusodon cf. quintuplinervis (Princ. Neuwied) Koehne, Ossaea cinnamomifolia Triana, Ossaea coriacea Triana, Trembleya laniflora var. genuina Cogn., Peixotoa sp., Irlbachia pedunculatus (Cham. \& Schlecht.) Gilg., Episcia sp., Escallonia cf. clorophylla Cham. \& Schlecht., Gaylussacia salicifolia Cham. \& Schlecht., Paepalanthus sp., Singonanthus sp., Oncidium sp., Fimbristylis autumanalis (L.) R. \& P., Melinis minutiflora Beauv., Andropogon bicornis L., Digitaria sp., Paspalum polyphyllum Nees, Panicum millegrana Poir., Erianthus sp. e Cuphea cf. disperma Koehne. No campo sujo de cerrado, onde já ocorrem em maior percentagem arbustos e árvores de pequeno, porte foram identificadas Jacaranda caroba (Vell.) DC. (jacarandazinho), Ilex cf. theezans Mart., Didymopanax macrocarpum (Cham. \& Schlecht.) Seem., Erythroxylum suberosum St. Hil., Baccharis platypoda DC., Eremanthus incanus Less. (candeião), Vanillosmopsis erythropappa Schul. Bip. (candeia), Stenocline sp. 
(marcela), Melinis minutiflora Beauv. (capim-gordura), Diplusodon cf. quintuplinervis (Princ.Weuwied) Koehne, Ciperáceas (Fimbristylis sp., Rynchospora spp.) e Gramíneas (Paspalum sp., Panicum sp., Digitaria sp., Erianthus sp.).

Áreas Úmidas/Brejos Pemanentes - A vegetação aquática ('macrófitas aquática') ocorre nas "áreas úmidas" que são "áreas de pântano, brejo, turfeira ou com água, naturais ou artificiais, permanentes ou temporárias, com água corrente ou parada, doce, salobra ou salgada, incluindo áreas marinhas, cuja profundidade na maré baixa não exceda seis metros"(IUCN 1988). Na E.E.T. existe uma lagoa artificial próxima à sede, vários córregos nas depressões e, a uma certa distância dos cursos d'água, brejos permanentes com solo higromórfico, cujo nível de inundação diminui no auge da estação seca (setembro a outubro). Algumas dessas áreas encontram-se em adiantado estádio sucessional, onde as espécies terrestres já ocupam papel significativo. Hedychium coronarium Koeng. (lírio-do-brejo), Sauvagesia erecta L., Commelina erecta L. (trapoeiraba), Begonia hispida Schott ex DC. (begônia), Tripogandra diuretica (Mart.) Handl., Drosera montana Mart., Juncus dichotomus Ell., Anemia phyllitidis (L.) Sw., Sticherus pruinosus (Mart.) Ching (samambaia), Blechnum serrulatum Rich., Centella biflora Nannf., Xyris sp. (botão-de-ouro), Syphocampylus convolvulaceus G. Don. e Ludwigia laruotteana (Camb.) Hara são algumas das espécies identificadas nesta formação.

Em muitas áreas, próximas aos cursos d'água perenes, observam-se florestas que necessitam ser melhor estudadas, para que se possa definir de forma mais clara a que tipo pertencem, pois se assemelham, fisionomicamente, às florestas mesófilas, às florestas de galeria, aos 'candeiais' e até mesmo às formações sucessionais.

Formações Sucessionais - Sucessões são processos ordenados de mudanças em uma comunidade vegetal que foi perturbada na sua composição florística e estrutura originais e nos solos sobre os quais estão estabelecidas (Ehrlich \& Roughgarden 1987). Mudanças climáticas, geológicas e a intervenção humana são os principais fatores causadores desta perturbação. Assim, as chamadas "capoeiras" que ocorrem na E.E.T. são formações sucessionais que podem evoluir e apresentar composição florística e estrutura semelhantes às formações pré-existentes. Nessas áreas, são comuns espécies herbáceas invasoras e espécies pioneiras, tais como Achryrocline satureoides (Lam.) DC. (marcela), Baccharis trimera DC. (carqueija), Abatia americana (Gardner) Eich., Hyptis membranacea Benth., Trembleya parviflora (Don.) Cogn., Gleichenia bifida (Willd.) Ching. (samambaia), Coccosypselum erythrocephalum Cham. \& Schlecht., Psychotria cf. sessilis (Vell.) Muell. Arg., Lantana lilacina Desf., Triumpheta semitriloba Jacq. e Geophila sp. (Tab. 1).

O "candeial" (Área 6-Trevo) se insere nas sucessões secundárias como uma formação pioneira de Vanillosmopsis erythropappa Schul. Bip., que se desenvolve após a perturbação da floresta mesófila, cujo número de indivíduos diminui gradativamente, à medida que a floresta atinge estádios mais avançados de desenvolvimento, na sua estrutura e composição florística. Quando as árvores da floresta mesófila ultrapassam em altura as da "candeia", competindo por luz e nutrientes, 
causando-lhes sombreamento cada vez maior, observa-se a diminuição do número de indivíduos desta espécie, até seu desaparecimento no interior da floresta.

As florestas mesófilas estacionais da E.E்.T., particularmente nas Áreas 1 - Pomar e 5- Esperto, apresentaram semelhanças florísticas e estruturais com outras florestas estudadas na região sudeste do Brasil, em Minas e São Paulo (Cavassan et al. 1984; Pagano \& Leitão Filho 1987; Vieira et al. 1989; Rodrigues et al. 1989; Vilela et al. 1994; Pagano et al. 1995).

A variação da deciduidade das florestas mesófilas na E.E.T. está relacionada à concentração dos componentes químicos dos solos, que apresentam baixos teores de $\mathrm{Ca}, \mathrm{Mg}$ e $\mathrm{P}$, altos teores de $\mathrm{Al}$ e acidez elevada (CETEC 1996). Este fato foi também observado por Ratter et al. (1978) ao estudarem florestas em solos mesotróficos no Brasil Central no Vale dos Sonhos (MT), Padre Bernardo (GO) e Januária (MG).

Apesar de fisionomicamente semelhantes, apenas duas das áreas estudadas apresentaram similaridade florística, o que está de acordo com o postulado por Whittaker (1977) para comunidades florestais em geral, que formam verdadeiros mosaicos, embora possam apresentar tipos dominantes de vegetação, como no caso da E.E.T., representado pelas florestas mesófilas.

\section{Agradecimentos}

À Fundação Estadual do Meio Ambiente de Minas Gerais - FEAM, pelo apoio logístico, principalmente na pessoa do administrador da E.E.T., o Biólogo Aristides Guimarães Salgado Neto e aos demais funcionários, pela colaboração nos trabalhos de campo; à Bióloga Yule Roberta Ferreira Nunes e aos técnicos em atividades de pesquisa Geraldo Pereira de Souza e José Carlos dos Santos (SAT/CETEC) pelo apoio nos trabalhos de campo; ao Prof. Dr. Waldir Mantovani, Departamento de Ecologia/ USP, pelas orientações e discussões com relação à definição da rede de amostragem; ao Prof. Dr. José Rubens Pirani, Profa. Maria Leonor del Rey Souza, Dr. Jefferson Prado e ao Pesquisador Luciano de Bem Bianchetti que auxiliaram na identificação taxonômica das plantas.

\section{Referências bibliográficas}

Ab' Saber, A.N. 1977. Os dominios morfoclimáticos na América do Sul. Boletim do Instituto de Geografia da Universidade de São Paulo 52:1-21.

Antunes, F.Z. 1986. Caracterização climática do Estado de Minas Gerais. Informações Agropecuárias 12: 913.

Araújo, G.M. 1992. Comparação da estrutura e do teor de nutrientes nos solos e nas folhas de espécies arbóreas de duas matas mesófilas semidecíduas no triângulo Mineiro. Campinas. Unicamp. Tese de Doutorado.

Araújo, L.C. 1944. Vanillosmospsis erythropappa (DC.) Sch. Bip. e sua exploração florestal. Rio de Janeiro. Escola Nacional de Agricultura. Tese para Professor Catedrático.

Badini, J. 1985. Flora do Tripuí: Anotações técnicas da UFOP. Ouro Preto. Departamento de Botânica/ UFOP. 6p. (poligrafo).

Baldini, J. 1988. Estação Ecológica do Tripuí e sua Flora: Anotações técnicas da UFOP. Ouro Preto. Departamento de Botânica/UFOP. 13p. (poligrafo).

Barroso, G.M.; Guimarães, E.F.; Ichaso, C.L.F.; Costa, C.G. \& Peixoto, A.L. 1978. Sistemática de Angiosperma do Brasil: I. São Paulo. LCT/EDUSP. 
Barroso, G.M.; Guimarães, E.F.; Ichaso, C.L.F.; Costa, C.G.; Peixoto, A.L. \& Lima, H.C. 1984. Sistemática de Angiosperma do Brasil: II. Viçosa. Imprensa Universitária.

Barroso, G.M.; Guimarães, E.F.; Ichaso, C.L.F.; Costa, C.G.; Peixoto, A.L. \& Lima, H.C. 1986. Sistemática de Angiosperma do Brasil: III. Viçosa. Imprensa Universitária..

Bertoni, J.E. A. \& Martins, F.R. 1987. Composição floristica de uma floresta ripária na Reserva Estadual de Porto Ferreira, SP. Acta Botanica Brasilica 1(1): 17-26.

Carvalho, D.A.; Oliveira-Filho, A.T.; Vilela, E.A. \& Gavilanes, M.L. 1995. Flora arbustivo-arbórea de uma floresta ripária no alto rio Grande em Bom Sucesso/MG. Acta Botanica Brasílica 9(2): 231-245.

Cavassan, O.; Cesar, O. \& Martins, F.R. 1984. Fitossociologia da vegetação arbórea da Reserva Estadual de Bauru, Estado de São Paulo. Revista Brasileira de Botânica 7 (2):91-106.

CETEC - FUNDAÇÃO CENTRO TECNOLÓGICO DE MINAS GERAIS. 1992. Ecofisiologia da 'candeia' (Vanillosmopsis sp.) visando seu aproveitamento ecológico e econômico. Belo Horizonte. SAT/CETEC. Relatório técnico.

CETEC - FUNDAÇÃO CENTRO TECNOLÓGICO DE MINAS GERAIS. 1996. Ecofisiologia da candeia. Belo Horizonte: SAT/CETEC. Relatório técnico.

Cook, C.D.K. 1974. Water plants of the world. The Hague, Dr. W. Junk b.v. Publ.

Costa, L.G.S. 1992. Estrutura e dinâmica de um trecho de mata mesófila semidecídua na Estação Experimental de Ibicątú, Piracicaba, SP. São Paulo. Universidade de São Paulo, Departamento de Ecologia. Dissertação de Mestrado.

Cronquist, A. 1988. The evolution and classification of flowering plants. New York. The New York Botanical Garden.

Durigan, G. 1994. Florística, Fitossociologia e produção de folhedo em matas ciliares da região oeste do Estado de São Paulo. Campinas. Universidade Estadual de Campinas. Departamento de Ecologia. Tese de Doutorado.

Ehrlich, P.R. \& Roughgarden, J.R. 1987. The science of ecology. New York. Macmillan. p. 5-90.

Eiten,G.1983. Classificação da vegetação do Brasil. Brasilia: CNPq.

FEAM - FUNDAÇÃO ESTADUAL DO MEIO AMBIENTE. 1995. Plano de Manejo da Estação Ecológica do Tripuí, Ouro Preto, MG. Belo Horizonte. FEAM. V.1.

Fernandes, A.G. \& Bezerra, P. 1990. Estudo Fitogeográfico do Brasil. Fortaleza: Stylus Comunicações.

Ferri, M.G. 1980. Vegetação Brasileira. Belo Horizonte. Itatiaia; São Paulo. EDUSP.

Font Quer, M.P. 1977. Diccionario de botánica. Barcelona: Lábor.

Gandolfi, S. 1991. Estudo florístico e fitossociológico de uma floresta residual na área do Aeroporto Internacional de São Paulo, Município de Guarulhos, SP. Campinas. Universidade Estadual de Campinas. Departamento de Botânica. Dissertação de Mestrado.

IBGE - FUNDAÇÃO INSTITUTO BRASILEIRO DE GEOGRAFIA E ESTATÍSTICA. 1992. Manual Téenico da Vegetação Brasileira. Rio de Janeiro. IBGE.

IUCN - UNIÃO PARA A CONSERVAÇÃO DA NATUREZA. 1988. Campanha para a proteção de áreas úmidas. Brasilia: IUCN/WWF/IBDF. 10p.

Leitão-Filho, H.F.; Azevedo, D.B.; Santin, D.A.; Gardolinski, P.C.F.C. \& Rodrigues, R.R. 1994. Estudos de ecologia da mata ciliar dos rios Mogi Guaçu e Peixe - UHE Mogi Guaçu - São Paulo. Campinas. Departamento Botânica/Unicamp.

Magurran, A.E.1988. Ecological diversity and its measurement. Princeton. University Press.

Mantovani, W. 1992. Fitossociologia. São Paulo. Departamento de Ecologia/USP. 28p. (poligrafo).

Martins, F.R. 1991. Estrutura de uma floresta mesófila. Campinas. Unicamp.

Martins, F.R. 1992. Métodos de amostragem em vegetação. Aracaju. UFSe/SBB, 10p. (poligrafo).

Martius, C.F.P., Eichler, G.F. \& Urban, I. (eds.). 1840-1906. Flora Brasiliensis Enumeratio Plantarum. Monachii. Lipsiae.16v., il.

Meguro, M.; Pirani, J.R.; Mello-Silva, R. \& Giulietti, A.M. 1996. Caracterização florística e estrutural de mata ripárias e capões de altitude da Serra do Cipó, MG. Boletim de Botânica da Universidade de São Paulo 15: 13-29.

Meira-Neto, J.A.A.; Bernacci, L.C.; Grombone, M.T.; Tamashiro, J.Y. \& Leitão-Filho, H.F. 1989. Composição floristica da floresta semidecídua de altitude do Parque Municipal da Grota Funda (Atibaia, SP.). Acta Botanica Brasilica. 3(2): 51-74. 
Mori, S.A., Mattos-Silva, L.A., Lisboa, G. \& Coradin, L. 1989. Manual de manejo do herbário fanerogâmico. $2^{a}$ ed. Ilhéus. CEPLAC.

Muller-Dombois, D. \& Ellenberg, H. 1974. Aims and methods for vegetation ecology. New York. J. Wiley \& Sons.

Oliveira-Filho, A.T.; Almeida, R.J.; Mello, J.M. \& Gavilanes, M.L. 1994. Estrutura fitossociológica e variávies ambientais em um trecho da mata ciliar do córrego dos Vilas Boas, Reserva Biológica do Poço Bonito, Lavras (MG). Revista Brasileira de Botânica 17(1): 67-85.

Pagano, S.N.; Leitão-Filho, H.F. \& Shepperd, G.J. 1987. Estudo fitossociológico em mata mesófila semidecidua no município de Rio Claro (Estado de São Paulo). Revista Brasileira de Botânica 10: 49-61.

Pagano, S.N. \& Leitão-Filho, H.F. 1987. Composição florística do estrato arbóreo de mata mesófila semidecidua, no município de Rio Claro (Estado de São Paulo). Revista Brasileira de Botânica 10: 37-47.

Pagano, S.N. 1989. Produção de folhedo em Mata Mesófila Semidecídua no Municipio de Rio Claro, SP. Revista Brasileira de Botânica 49(3): 633-639.

Pagano, S.N.; Leitão-Filho, H.F. \& Cavassan, O. 1995. Variação temporal da composição florística e estrutura fitossociológica de uma floresta mesófila semidecidua, Rio Claro, Estado de São Paulo. Revista Brasileira de Biologia 55(2): 241-58.

Ratter, J.A.; Askew, G.P.; Montgomery, R.F. \& Gifford, D.R. 1978. Observations on forests of some mesotrophic soils in central Brazil. Revista Brasileira de Botânica 1:47-58

Reitz, R. 1965-1989. Flora Ilustrada Catarinense. Itajai, (SC), HBR/CNPq/U.S.Nat.Sc. Foudation/IBDF/ EMPASC. 161v., 12489p.

Rizzini, C.T. 1979. Tratado de fitogeografia do Brasil. São Paulo. HUCITEC/EDUSP.

Rodrigues, R.R.; Morellato, L.P.C.; Joly, C.A. \& Leitão-Filho, H.F. 1989. Estudo florístico e fitossociológico em um gradiente altitudinal de mata estacional mesófila semidecidua, na Serra do Japi, Jundiai, SP. Revista Brasileira de Botânica 12: 71-84.

Rodrigues, R.R. 1986. Levantamento florístico e fitossociológico das matas da Serra do Japi, Jundiaí, SP. Campinas. Universidade Estadual de Campinas. Departamento de Ecologia. Dissertação de Mestrado.

Rozza,A.F. \& Ribeiro, C.A. s.d. Estudo florístico e fitossociológico de fragmento de mata ciliar dos campos da ESALQ, Piracicaba, SP. Anais do VIII Congresso da Sociedade Botânica de São Paulo, p. 7-25.

Schiavini, I. 1992. Estrutura das comunidades arbóreas de mata de galeria da Estação Ecológica dos Panga (Uberlândia, MG). Campinas. Universidade Estadual de Campinas. Departamento de Ecologia. Dissertação de Mestrado.

Tomasulo, P.L.B. 1995. Análise da composição florística e estrutura da vegetação como subsídio ao plano de manejo do Parque Municipal da Serra do Itapety, Mogi das Cruzes, São Paulo. Belo Horizonte. Universidade Federal de Minas Gerais. Departamento de Ecologia Geral. Dissertação de Mestrado.

Veloso, H.P. 1966. Atlas Florestal do Brasil. Rio de Janeiro. Ministério da Agricultura.

Vieira, M.G.L.; Moraes, J.L.; Bertoni, J.E.A.; Martins, F.R. \& Zandarin, M.A.1989. Composição florística e estrutura fitossociológica da vegetação arbórea do Parque Estadual de Vaçununga, Santa Rita do Passa Quatro (SP). II- Gleba Capetinga Oeste. Revista do Instituto Florestal 1(1): 135-59.

Vilela, E.A.; Oliveira-Filho, A.T.; Carvạlho, D.A. \& Gavilanes, M.L. 1994. Fitossociologia e fisionomia de mata semidecidua margendo o reservatório de Camargos em Itutinga, Minas Gerais. Ciência e Prática 18(4):415-24.

Whittaker, R.H. 1977. Classification of natural communities. New York. Arnopress. 